\title{
Research on new remote terminal unit transmission scheme based on distributed deployment
}

\author{
Shanqiang Feng, Xiaojun Chen, Kai Ma \\ Electric Power Research Institute of Guangdong Power Grid Corporation, Guangzhou, Guangdong, \\ 510080, China; \\ fengshanqiang@139.com
}

Keywords: IEC 61850; distributed technology; new RTU, source end maintenance; intelligent alarming

Abstract. The current smart grid architecture, which has two-level data centers and two-level operation applications, is faced with many deficiencies. In order to solve these problems, this paper puts forward a distributed system architecture based on IEC 61850 remote terminal unit (RTU) data transmission scheme. According to the architecture interested, a distributed deployment of the automated operations was realized at the master station and the slave station, and distributed modeling was performed for the operation data, which were then transmitted according to IEC 61850. Then, several key problems about the RTU transmission scheme were discussed, including function division for the master station and the slave station, RTU modeling and source end maintenance.

\section{Introduction}

At present, the whole power industry around the world is faced with a tide of exploration and research in view of the rapid development of technologies, including microchip, optical communication and software architecture. Under the guidance of the technical specification of main station integration, China's power grid is developing at a steady pace in the field of power automation, and making great progress. For the construction of a station, the station-end automation providers have been able to provide a panoramic data center integrating process level, bay level and station level, based on IEC 61850. This data center not only provides the supervising and control functions as the conventional station-end SCADA, but also continuously explores how to use different advanced operation applications at the data source end of the power grid, which applications are usually used at the master station, for example, in-station zero-impedance state estimation, distributed VAR compensation control, intelligent alarming and remote browsing.

In view of the rapid development of technologies and operations, it is necessary to take a serious look at this architecture with two-level data centers and two-level operation applications, for the purpose of a more reliable and safer architecture of the whole power grid system. According to literatures [1-4], some put forward new application modes for different advanced application services based on the conventional architecture, and some went even further by putting forward a new three-level architecture of the main station, while neither involved RTU transmission technology. Based on IEC 61850 (Edition 2) and the concept of distributed intelligent application, the paper puts forward a new scheme of distributed system architecture, and discusses the technology about using RTU as a transparent channel.

\section{System architecture analysis}

Current system architectures of master station and slave station.The Operation Smart System suggested in China Southern Power Grid OS2 is composed of master systems and slave systems at all levels. The new RTU at the station end serves for the comprehensive acquisition, intelligent preprocessing and unified access of all kinds of data within the station, which mainly include SCADA of monitoring equipment,information protection slave station, metering, state monitoring and PMU under supervision; supports the data exchange with the intelligent data center with the master; and 
supports the subscription and publication of data service. For the transmission protocol, the extended 104 protocol is adopted. As at two different levels, the slave data center and the master data center are independent relatively, and there is basically no complementary relation between the data analysis operations on these different data platforms, resulting in partial data redundancy and partial business overlay. For a smart substation, the new RTU acquires the MMS messages from the in-station intelligent IEDs, or gives control commands to the controlled IED devices, and the data relation between the slave data center and the RTU is not clear. At present, as IEC 61850 technology and smart substation become popular, additionally, as unattended substation becomes normal, the mass information of the substation is aggregated at the master data center, and there come more and more professional data analysis operations with the approach of the era of big data. The data centers undertake quite a lot of work load, and at the same time, the excessive centralization of data puts higher requirements for the stability, disaster tolerance and data scheduling of the systems, which make them overburdened.

Distributed system architecture based on IEC 61850. There is a precondition for the system architecture scheme presented in this paper, that the communication network composed of the new RTU and the master front-end client shall meet the requirement for the capacity of information transmission, and the current communication technology is able to provide such communication channel $^{[3]}$.

The new RTU is a special intelligent IED, with clients and server supporting IEC 61850 protocols. The clients exchange MMS messages with the in-station IEDs, and the server exchange MMS massages with the master front-end client. In the gateway, there is a rapid data exchange mechanism between the client and the server, to support the data forwarding between the master station and the slave station.

At the slave station, there is no change to the relation between the data center/new RTU and the in-station intelligent IEDs, and the data center and the new RTU exchange information with intelligent IEDs through the in-station data busbar. In the station, each data operation module is designed as a virtual intelligent IED, where the calculation output, in addition to being stored in the in-station data center, is transmitted to the new RTU through MMS messages as other in-station IEDs do, for data exchange with the operations of the same kind at the master station.

At the master station, the front-end client analyzes the RTU messages, inputs the data into the master data center, and at the same time, receives operation requests from the master station, and forwards to the slave station. If a client has adequate communication capability and channel capacity, it can communicate with a number of new RTUs. But for PMU, due to the large data volume and the high real-time performance, a dedicated RTU is adopted to transmit the data to the master station. Refer to Fig. 1 for the system structure.

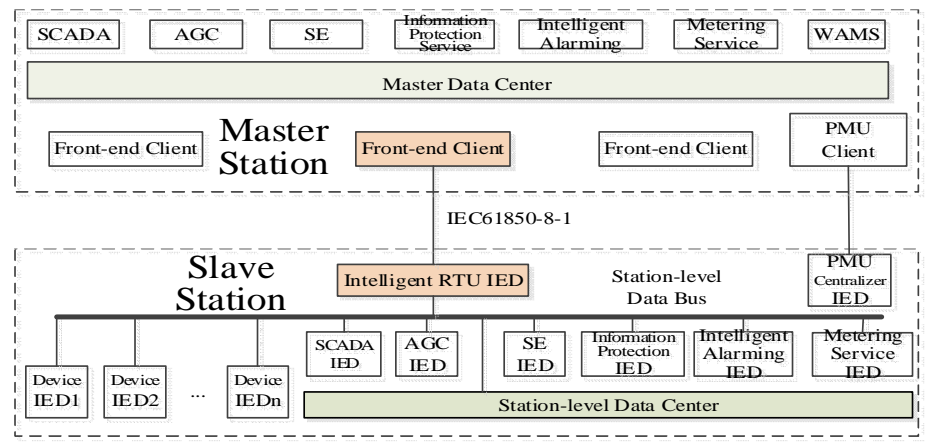

Fig.1 System structure 


\section{Key technologies of new RTU}

Function division for master station and slave station. The functions of the master front-end client include: source end maintenance for master station; MMS message analysis and updating the data of CIM model; receiving the service requests for master station application (e.g. remote control, HV data monitoring, metering, power quality inspection, fixed value management) and forwarding to RTU; file receiving, unzipping, caching, etc.

The functions of the new RTU: source end maintenance for slave station; receiving MMS messages from intelligent IEDs at the slave station, and re-integrating the messages to be mapped to new logical devices; forwarding the service requests from the master station to the in-station devices; file zipping and transferring to the master station. The functions to be completed with the master front-end: communication data redundancy, access safety control, data channel maintenance.

New RTU modeling. The physical device of the new RTU could be a device with multiple embedded CPUs, or a workstation. For the inside of the station, it is a client taking the in-station SCD file as the model, which is able to receive the messages from all the intelligent IEDs (including virtual devices), and forward data and commands to these IEDs; on the other side, for the master station, it is a server integrating the logic nodes of all the intelligent devices across the station, forming a station-level intelligent IED. According to IEC 61850, the server of the intelligent IEDs has four attributes, namely logic device, access point, file and client correlation. Only the data model of the logic device requires for maintenance, and other attributes are standard services. This model requires for re-construction with source end maintenance technology.

Each IED at the Smart Substation might comprise one or more logic devices, and a new logic device is constructed to describe the new RTU itself. The RTU model might simply comprise the logic devices of all the IEDs in the station and the new constructed virtual logic devices of different advanced operations, as shown in Fig. 2.

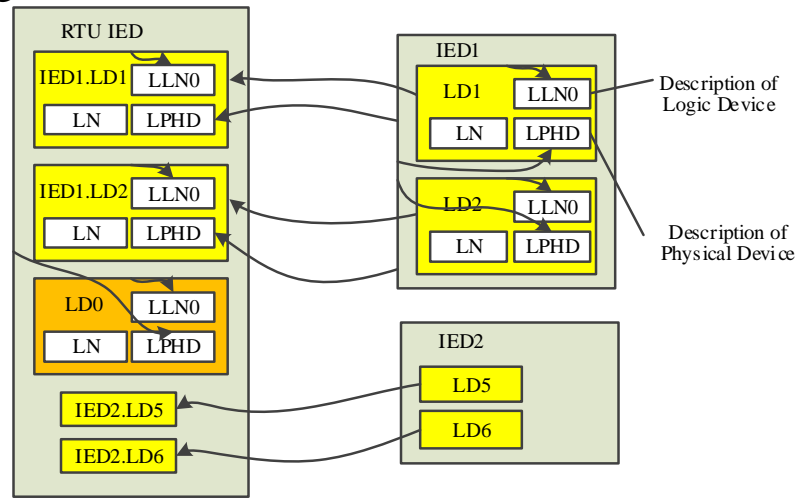

Fig.2 RTU model

Just like LD1 in IED1, each logic device must comprise LLN0 and LPHD. LPHD describes the information of the physical device of IED1, including the information on the nameplate of the device, even the data space of the device and so on; LLNO comprises the data sets of the logic device, the control blocks of correlated services and so on, which describes the logic device LD1. In the RTU model, an essential logic device LD0 is added, to describe the gateway itself. Almost all the LDs of IED1 and IED2 are migrated into the new model, and there is no change to LPHD or LN, while the data sets for LLN0 in the new model to transmit the data and the control blocks of correlated services change according to the demand for source end maintenance. Each logic node completely exposes to the master station, and the master station is able to query the data of any logic node according to the need and authority, or to perform remote control.

Source end maintenance technology. The conventional source end maintenance files are mainly CIM, SVG and MAP files, so that after these files have been used to maintain and modify the data, models and graphics at the salve station, model files that the master station can access in a seamless manner are provided automatically, which means that the files can be shared by the master stations at all levels after 
maintenance ${ }^{[4]}$. Since this technology is still in the exploration and development phase, the maintenance model is applied to the "four remote" information of the master SCADA, namely remote measurement, remote signal, remote control and remote regulation. When the distributed application scheme presented in the paper is applied, the content to be maintained shall be extended; the master station uses the SCD files of IEC 61850 to acquire the data; and there would be changes to the files to be maintained, as well as to the methods.

Since the master station uses the main SCD files generated at all the station-level IEDs, the master station has the ability to analyze the SCD files, and the CIM files that used to be generated at the slave station now are generated at the master station in a unified way; at the same time, there are some changes to the content of the MAP files that used to be needed at the master station: 1) the data needed by the master maintenance objects are selected from the DOs of each IED in the SCD files, to form the data set; and 2) requirements are made for data transmission, for the slave station to restrain the data transmission.

The content of maintenance is as follow: 1) to select the data with the MAP files at the master station, to generate the data set of the station-level IEDs of the RTU; 2) to apply the data restraint of MAP to complete the automatic generation of the control blocks; 3) to generate the mapping relation between the SVG graphic elements and the data in the data set, for the master station to correlate the graphics with the data.

\section{Conclusions}

In view of the new situation presented by the rapid development of the main station integrated system supervising system, the paper put forward a distributed system architecture based on the two levels of full scenario data centers at the master station and the slave station, taking into consideration the specific condition of the integrated supervising system of China Southern Power Grid. Then, the key communication technologies of the distributed system architecture were researched respectively, and a new function division was performed to the communication modules of the master station and the slave station; according to IEC 61850, the slave RTU was modeled, and a solution different than the traditional method was put forward for the source end maintenance technology. Finally, a virtual IED modeling scheme was presented for the advanced operations that have been extensively researched in the field of distributed application, such as intelligent alarming and state estimation. Generally, the new RTU scheme presented in the paper is technically feasible, and is able to improve the reliability of the integrated supervising system, to reduce the pressure of the rapid expansion of the data volume at the master station, and to improve the disaster tolerance of the system, so that it is possible to use the large amount of redundant data at both the master station and the slave station in an efficient way, indicating a promising prospect of application.

\section{References}

[1] ZHU Yongli, HUANG Min, LIU Peipei, et al. Research on transmission of power telecontrol information based on IEC61850 and network[J]. Relay, 2005, 33(11): 45-49.

[2] ZHU Yongli, WANG Dewen, WANG Yan. A telecontrol communication modeling method based on IEC61850 [J]. Automation of Electric Power Systems, 2009, 33(21): 72-76.

[3] ZHANG Daojie, WANG Guangmin, et al. Research and application of integration of network protection dispatching and control[J]. Power System Protection and Control, 2013, 41(14): 149-153.

[4] HUANG Kai, YANG Ji, GU Quan. A source-based maintenance technology for integrated intelligent operation system of power grids[J]. Automation of Electric Power Systems, 2014, 38(15): $71-75$. 